\title{
Factores significativos del rendimiento excelente: PISA y otros estudios
}

\author{
Carmen Jiménez Fernández* \\ María de los Ángeles Baeza Delgado**
}

\section{Resumen}

Se centra en tres investigaciones sobre los alumnos con rendimiento excelente. El estudio de PISA Top of de class, que analiza los factores subyacentes al rendimiento excelente de los alumnos de 15-16 años; el estudio de una muestra de alumnos españoles que han obtenido Premio Extraordinario de Bachillerato (PEB) y un tercer estudio sobre alumnos que han obtenido Beca de Excelencia (BE)para estudiar en la universidad de Madrid. Se concluye que aunque la procedencia social no es determinante, la mayor parte de los alumnos con rendimiento excelente proceden de ambientes culturalmente favorecidos si bien el centro escolar ejerce mayor influencia que la clase social; la cantidad de horas semanales que el currículo dedica a la enseñanza de las materias fundamentales y la cantidad de horas que el alumno dedica al estudio son factores importantes del rendimiento excelente; los centros con mejor rendimiento atraen más a los alumnos con rendimiento excelente $y$, a su vez, hay más alumnos con rendimiento excelente en los centros más elitistas; en el estudio de PISA no hay diferencias de género; en los estudios sobre alumnos con PEB y BE persisten reductos sexistas.

Palabras clave: Factores del rendimiento excelente. Escuela y rendimiento excelente. Esfuerzo y rendimiento excelente.

\section{Fatores significativos no rendimento excelente: PISA e outros estudos}

\section{Resumo}

0 presente trabalho se centra em três pesquisas sobre alunos com rendimento excelente. 0 estudo de PISA Top of de class, que analisa os fatores subjacentes ao rendimento excelente de alunos entre 15 e16 anos; o estudo de uma amostra de

\footnotetext{
* Doctora en Filosofía y Letras por la Universidad Complutense de Madrid (España). Catedrática de Pedagogía Diferencial en la Universidad Nacional de Educación a Distancia (UNED). Facultad de Educación. E-mail: mjimenez@edu.uned.es.

** Catedrática del Instituto de Educación Secundaria “Altair”. Getafe (Madrid). E-mail: baezadel@gmail.com.
} 
alunos espanhóis que obtiveram o Premio Extraordinário de Ensino Médio e um terceiro estudo sobre alunos que obtiveram Bolsa de Excelência para estudar na Universidade de Madrid. Chega-se à conclusão que embora a procedência social não seja determinante, a maior parte dos alunos com rendimento excelente procedem de ambientes culturalmente favorecidos, ainda que o centro escolar exerce maior influência que a classe social; a quantidade de horas semanais que o currículo dedica ao ensino das disciplinas fundamentais e a quantidade de horas que o aluno dedica ao estudo são fatores importantes do rendimento excelente; os centros com melhor rendimento atraem mais aos alunos com rendimento excelente e, por sua vez, há mais alunos com rendimento excelente nos centros mais elitistas; no estudo de PISA não há diferenças de gênero, no Ensino Médio e na Universidade persistem redutos sexistas.

Palavras-chave: Fatores do rendimento excelente. Escola e rendimento excelente. Esforço e rendimento excelente.

\section{Significant factors in the yield excellent: PISA and other studies}

\section{Summary}

The article focuses on three pieces of research on students with excellent achievement. The PISA study Top of de class, which analyzes the underlying factors of excellent achievement in 15-16 years old students; a study of a sample of Spanish students who have obtained Extraordinary Baccalaureate Prize, and a third study of students who have obtained an Excellence Grant to study in University in Madrid. All three conclude that although social background is not determinant, most students with excellent achievement come from culturally favoured environments even though school plays a more important role than social class; the number of weekly hours the curriculm devotes to fundamental subjects as well as the number of hours the student devotes to personal study are important factors of excellent achievement; school centers with better achievement attract more students with excellent achievement and, furthermore, there are more students with excellent achievement in more elitist schools; in the PISA study there are no gender differences, in baccalaureate and and university sexist redoubts persist. Keywords: Factors of excellent achievemet. School in excellent achievement. Effort and excellent achievement.

\section{Planteamiento del tema}

Datos recientes de EUROSTAT señalan que en España el 31,2\% de los jóvenes entre 18 y 24 años ha abandonado los estudios sin finalizar la ESO; la media europea se sitúa en el $14,4 \%$. Si tomamos los informes de PISA como referente se aprecia que en España en las cuatro evaluaciones habidas $(2000,2003,2006,2009)$ y en los tres tipos 
de competencias medidas (matemática, científica, lingüistica), los estudiantes españoles de 15 años se sitúan varios puntos por debajo de la media de los países de la OCDE y tienen especial dificultad para formar parte de los alumnos con rendimiento elevado y excelente o categorías 5 y 6 en la escala de PISA. El desarrollo del talento es un objetivo prioritario de las sociedades que aspiran a liderar el conocimiento, la innovación y el desarrollo social, como ocurre con la Unión Europea.

En estas líneas se reflexiona sobre las caracteristicas personales, familiares e institucionales que parecen concurrir en el grupo de los alumnos que obtienen rendimiento excelente. Nos basamos en dos tipos de datos. De una parte, en los estudios PISA y particularmente en el informe Top of the class (2009). De otra, en la investigación realizada sobre dos grupos de alumnos con rendimiento excelente elegidos por haber obtenido Premio Extraordinario de Bachillerato y Beca de Excelencia, respectivamente. La consideración de ambos tipos de estudios permiten avanzar algunas ideas que ilustran qué hay detrás del rendimiento excelente y pueden ayudar la toma de decisiones en la política y práctica escolar cara a desarrollar una educación que sea al mismo tiempo excelente y equitativa.

\section{Los estudios PISA}

El rápido avance tecnológico y la demanda in crescendo de trabajadores altamente cualificados, ha conducido a un interés creciente por el desarrollo del talento considerado un recurso crítico para el avance científico, tecnológico y social. En la sociedad del conocimiento el capital humano es la mayor fuente de riqueza de un país y los países desarrollados, que aspiran a liderar dicha sociedad, son conscientes de que las personas muy cualificadas casi determinan el crecimiento económico y el desarrollo social. En este contexto, educar para la excelencia se convierte en un importante objetivo de política educativa y social. PISA lo explica claramente: mientras que las competencias básicas son importantes para que las nuevas tecnologias sean aprendidas, las competencias de alto nivel son críticas para la creación de nuevo conocimiento, de tecnología e innovación.

PISA, programa promovido por la Organización para el Desarrollo Económico y Social (OCDE), viene aplicándose cada tres años iniciando su andadura en el año 2000. Va dirigido a los estudiantes de 15 años de edad y se aplica a los países de la OCDE y a otros que se suman a la iniciativa para conocer los resultados del propio sistema escolar en el marco creado por PISA. En cada evaluación enfoca predominantemente un área específica: lectura en 2000, matemáticas en 2003, ciencias en 2006 y lectura en 2009. En 2012 se centrará en matemáticas y en el 2015 en ciencias. Su finalidad es ofrecer un panorama sobre las diferentes maneras en que los jóvenes desarrollan y aplican su pensamiento.

Establece seis niveles de rendimiento (realmente siete si se tienen en cuenta de forma independiente los alumnos del nivel 0 o alumnos que prácticamente desconocen las cuestiones planteadas en las pruebas). Por ejemplo en PISA 2006, centrado en las ciencias, los niveles son: 
- Nivel 1 - Estudiantes con un limitado conocimiento científico;

- Nivel 2 - Estudiantes que poseen habilidades para investigar de manera sencilla;

- Nivel 3 - Son capaces de identificar claramente los usos científicos en los contextos y aplicaciones generales;

- Nivel 4 - Son capaces de dirigir fenómenos específicos, haciendo inferencias acerca de la ciencia y la tecnología, y son capaces de tomar decisiones a partir del conocimiento científico y de la evidencia;

- Nivel 5 - Pueden identificar los componentes de muchas situaciones de vida complejas aplicando tanto conocimiento como conceptos científicos para cada una de estas situaciones, así como comparar, evaluar y seleccionar evidencia científica que responda a las situaciones cotidianas;

- Nivel 6 - Pueden identificar, explicar y aplicar consistentemente el conocimiento científico y el conocimiento acerca de la ciencia en una gran variedad de situaciones de vida complejas, y pueden vincular diferentes fuentes de información para justificar sus decisiones.

\section{Resultados generales de los Informes PISA}

En los cuatro informes habidos entre 2000 y 2009 y en los tres tipos de competencias medidas, el rendimiento medio de los alumnos españoles es inferior al de la media de los países de la OCDE, oscilando la diferencia entre 7 y 31 puntos en el caso de la competencia lectora, entre 15 y 24 puntos en el caso de la competencia matemática y entre 9 y 13 puntos en el caso de la competencia científica (Tabla 1). Estos informes han hecho aflorar la sensibilidad social y han puesto nuevamente sobre la mesa la cuestión de cómo lograr que la escuela conjugue equidad y excelencia.

Tabla 1- Resultados generales de los Informes PISA

\begin{tabular}{|c|c|c|c|c|c|c|}
\hline & \multicolumn{2}{|c|}{$\begin{array}{l}\text { Comprensión } \\
\text { Lectora }\end{array}$} & \multicolumn{2}{|c|}{$\begin{array}{l}\text { Competencia } \\
\text { Matemática }\end{array}$} & \multicolumn{2}{|c|}{$\begin{array}{l}\text { Competencia } \\
\text { Científica }\end{array}$} \\
\hline & $\begin{array}{l}\text { M e d i a } \\
\text { España }\end{array}$ & $\begin{array}{l}\text { M e d i a } \\
\text { OCDE }\end{array}$ & $\begin{array}{l}\text { M e d i a } \\
\text { España }\end{array}$ & $\begin{array}{l}\text { M e d i a } \\
\text { OCDE }\end{array}$ & $\begin{array}{l}\text { M e d i a } \\
\text { España }\end{array}$ & $\begin{array}{l}\text { M e d i a } \\
\text { OCDE }\end{array}$ \\
\hline PISA 2000 & 493 & 500 & 476 & 500 & 491 & 500 \\
\hline PISA 2003 & 481 & 494 & 485 & 500 & 487 & 500 \\
\hline PISA 2006 & 461 & 492 & 480 & 498 & 488 & 500 \\
\hline PISA 2009 & 481 & 493 & 483 & 496 & 488 & 501 \\
\hline
\end{tabular}

Fonte: Elaboración propia a partir de los datos de los cuatro Informes PISA citados en las referencias bibliográficas'.

1 Los datos de los resultados de PISA 2000 han sido tomados de Resultados en España del estudio PISA 2000. Conocimientos y destrezas de los alumnos de 15 años. Los correspondientes a 2003 de la publicación de Ed. Santillana. Los de 2006 y 2009 se han tomado de la base de datos PISA 2006 y PISA 2009 en la web de la OCDE citadas en las referencias bibliográficas. 


\section{Resultados de los alumnos con rendimiento excelente}

Al considerar solamente a los alumnos con rendimiento excelente o top perfomers, se repite la situación anterior pues el promedio de los alumnos españoles con rendimiento excelente es inferior a la media de los alumnos de la OCDE, y ello en las cuatro evaluaciones habidas y en las tres áreas. La diferencia oscila entre 3,30 y 6,77 puntos porcentuales en el caso de la lectura, entre 4,74 y 6,80 puntos porcentuales en el caso de las matemáticas y entre 4,10 y 4,54 en el caso de ciencias (Tabla 2). Por ejemplo, en Competencia Matemática en 2003 el porcentaje de alumnos españoles con rendimiento excelente es 6,8 puntos inferior que el de la OCDE, 6 puntos inferior en el caso de 2006 y 4,74 inferior en 2009.

Para entender bien la Tabla 2 hay que señalar que en Lectura solo hay cinco niveles y se ha considerado Top Performers o con rendimiento excelente a los que alcanzan el nivel 5. En Matemáticas y Ciencias hay 6 niveles y se han considerado Top Performers a los que alcanzan el nivel 5 sumado a los que alcanzan el nivel 6. En PISA 2000 solo hay escala por niveles en Lectura; en 2003 hay dos escalas Lectura y Matemáticas y en 2006 quedan establecidas tres escalas o Lectura, Matemáticas y Ciencias.

Tabla 2 - Resultados de los alumnos Top Performers en los informes PISA

\begin{tabular}{l|l|l|l|l|l|l}
\hline & \multicolumn{2}{|l}{ Comprensión Lectora } & \multicolumn{2}{l|}{$\begin{array}{l}\text { Competencia } \\
\text { Matemática }\end{array}$} & \multicolumn{2}{l}{$\begin{array}{l}\text { Competencia } \\
\text { Cientifica }\end{array}$} \\
\cline { 2 - 7 } & España & OCDE & España & OCDE & España & OCDE \\
\hline PISA 2000 & $4 \%$ & $9 \%$ & No escala & No escala & No escala & No escala \\
\hline PISA 2003 & $4,98 \%$ & $8,28 \%$ & $7,9 \%$ & $14,7 \%$ & No escala & No escala \\
\hline PISA 2006 & $1,79 \%$ & $8,56 \%$ & $7,2 \%$ & $13,4 \%$ & $4,89 \%$ & $8,99 \%$ \\
\hline PISA 2009 & $3,18 \%$ & $6,77 \%$ & $8,04 \%$ & $12,7 \%$ & $3,96 \%$ & $8,5 \%$ \\
\hline
\end{tabular}

Fonte: Elaboración propia a partir de los datos de los cuatro Informes PISA 2 .

El Gráfico 1 muestra los resultados en matemáticas por niveles de competencia en los estudios PISA 2003 y 2006. La escala de matemáticas se compone de 6 niveles de competencia y los niveles 5 y 6 corresponden a los Top Performers; el nivel 4 corresponde a los Strong Performers. En España la media de los dos grupos, Top y Strong, se sitúa por debajo de la media de la OCDE, habiendo un 6,8\% y 6,2\% más de alumnos Top en la OCDE que en España.

2 Los datos de los resultados de PISA 2000 han sido tomados de Resultados en España del estudio PISA 2000. Conocimientos y destrezas de los alumnos de 15 años. Los correspondientes a 2003 de la publicación de Ed. Santillana. Los de 2006 y 2009 se han tomado de la base de datos PISA 2006 y PISA 2009 en la web de la OCDE la OCDE citadas en las referencias bibliográficas. 
Gráfico 1 - Resultados MATEMÁTICAS grupos Strong y Top en PISA 2003 y 2006

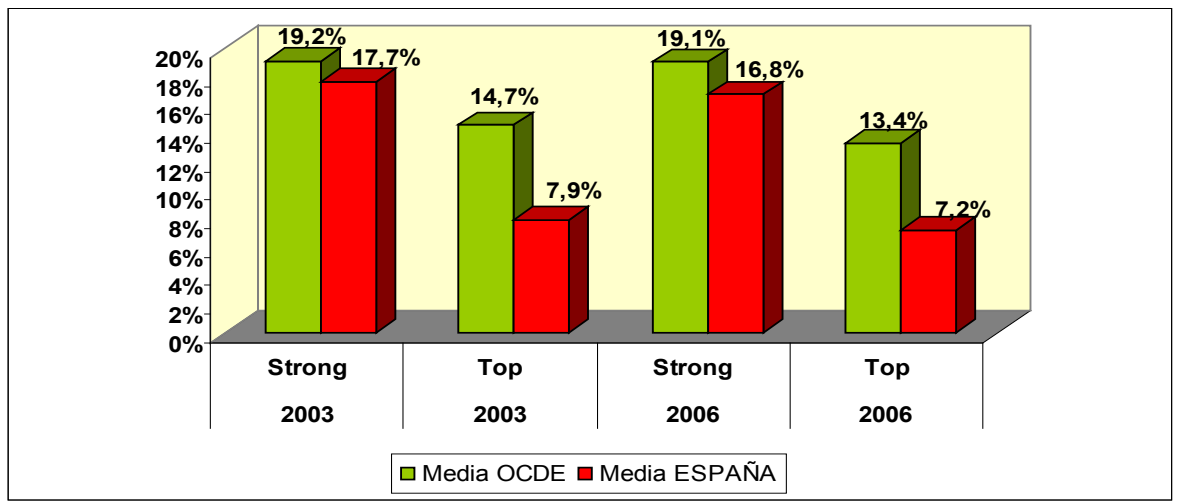

Fonte: OCDE. Base de datos PISA 2003 y 2006.

Un último ejemplo. Los Gráficos 2 y 3, del informe de 2009, muestran que el porcentaje de alumnos españoles en la competencia científica en la categoría 5 es tres puntos inferior al promedio de la OCDE y cuatro puntos inferior en la competencia lingüistica, y que en ambos tipos de competencias España carece de alumnos excelentes en la categoría 6. Resultados que llevan a preguntarse si es que nuestro país no tiene alumnos capaces del más alto rendimiento o es que se los traga el sistema.

Gráfico 2 - Distribución del rendimiento de los alumnos de ESO en los niveles de la competencia científica

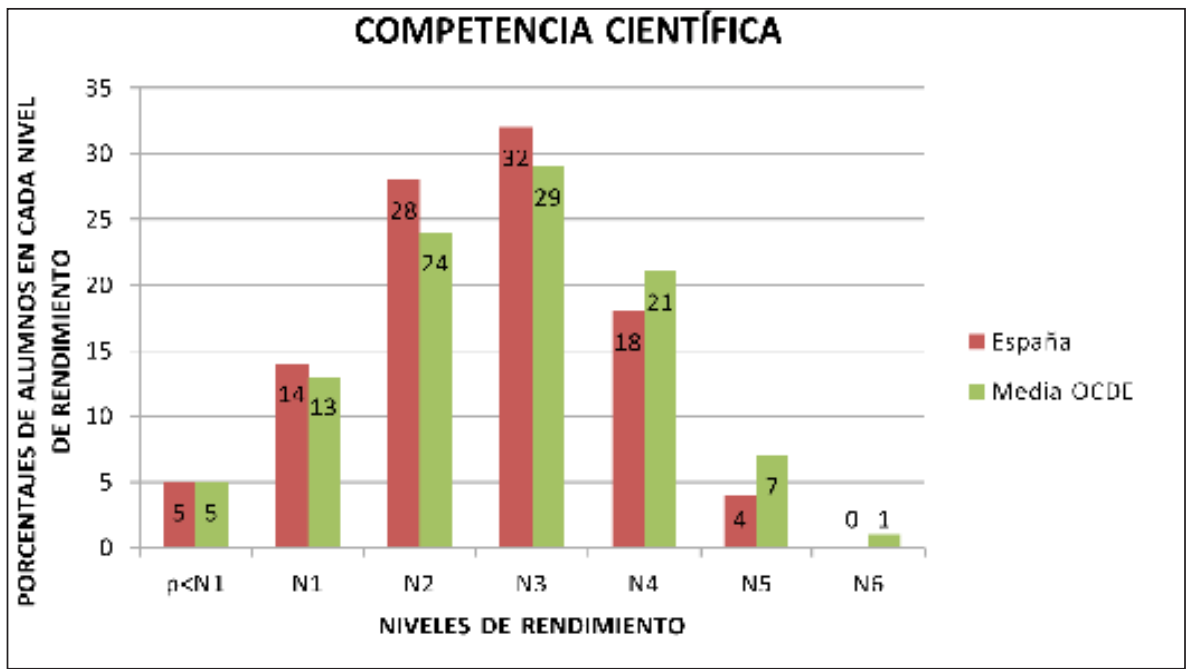

Fonte: PISA. PISA 2009: results: What makes a School successful? Resources, Policies and practices. OCDE, 2010. 
Gráfico 3 - Distribución del rendimiento de los alumnos de ESO en los niveles de la competencia lingüística

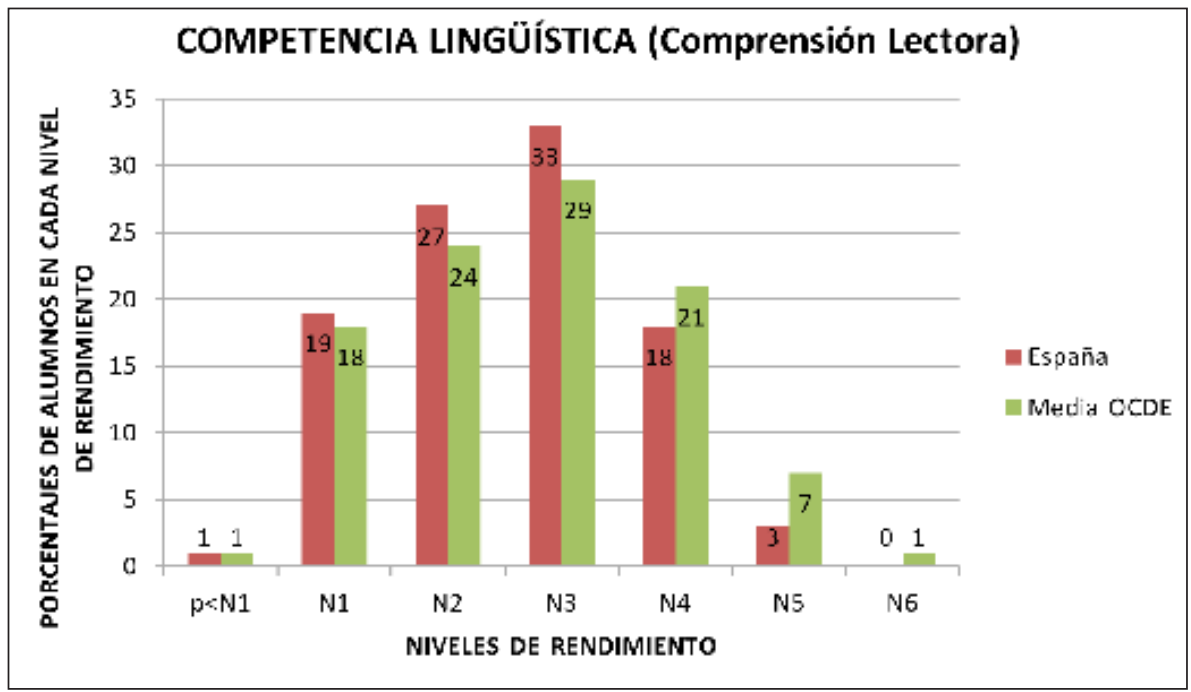

Fonte: PISA. PISA 2009: results: What makes a School successful? Resources, Policies and practices. OCDE, 2010.

La conclusión es que en ninguno de los cuatro informes y en ninguna de las tres competencias evaluadas logran los alumnos españoles tener un subgrupo con rendimiento excelente de tamaño similar a la media de los alumnos de la OCDE. Nuestra escuela produce alumnos con rendimientos mediocres. ¿Qué características parecen asociadas a los sistemas escolares y al grupo de alumnos con rendimientos excelentes?

\section{El informe Top of the Class o las variables que inciden en el alto rendimiento}

En 2006 los técnicos de PISA estudiaron con cierto detenimiento qué características parece tener la educación que recibe la población con rendimiento excelente explorando nuevos tipos de datos. El informe Top of the Class nace con este objetivo e incorpora a la evaluación factores relacionados con las características de los estudiantes o quienes son, el tipo de familias y comunidades a las que pertenecen, las características de los centros a los que asisten, su experiencias docentes en ciencias, la medida en que realizan actividades relacionadas con las ciencias fuera de la escuela, sus motivaciones para estudiar ciencias, su actitud hacia la ciencia y sus intenciones de realizar carreras relacionadas con las ciencias, a fin de clarificar el cómo del rendimiento excelente. Recordemos que el Informe 2006 tuvo la competencia científica como principal tema y por ello los cuestionarios aplicados indagan especialmente sobre ella. Top of the Class clasifica a los alumnos en cuatro categorias: 
- Top Performers o alumnos con rendimiento excelente. Se definen como aquellos que han puntuado en los dos niveles más altos de competencia 0 niveles 5 y 6 en la escala de ciencias. Corresponde a una puntuaron por encima de 633.33 en dicha escala;

- Strong Performers o alumnos con rendimiento elevado. Corresponden a los del nivel 4 y su puntuación está por encima de 558,7;

- Moderate Performers o alumnos con rendimiento moderado. Corresponden a los niveles 2 y 3 de la escala y puntuación por encima de 409,5;

- Lowest Performers o alumnos con rendimiento moderadamente bajo. Corresponden al nivel 1 o por debajo de éste y puntuaciones por encima de 334,9.

En el Gráfico 4 se observa una correlación positiva entre la proporción de alumnos que han puntuado en los niveles 5 y 6 de la escala PISA y el número de investigadores por cada mil personas empleadas en el país.

Gráfico 4 - Top performers en ciencias en PISA 2006 y magnitud de la investigación del país

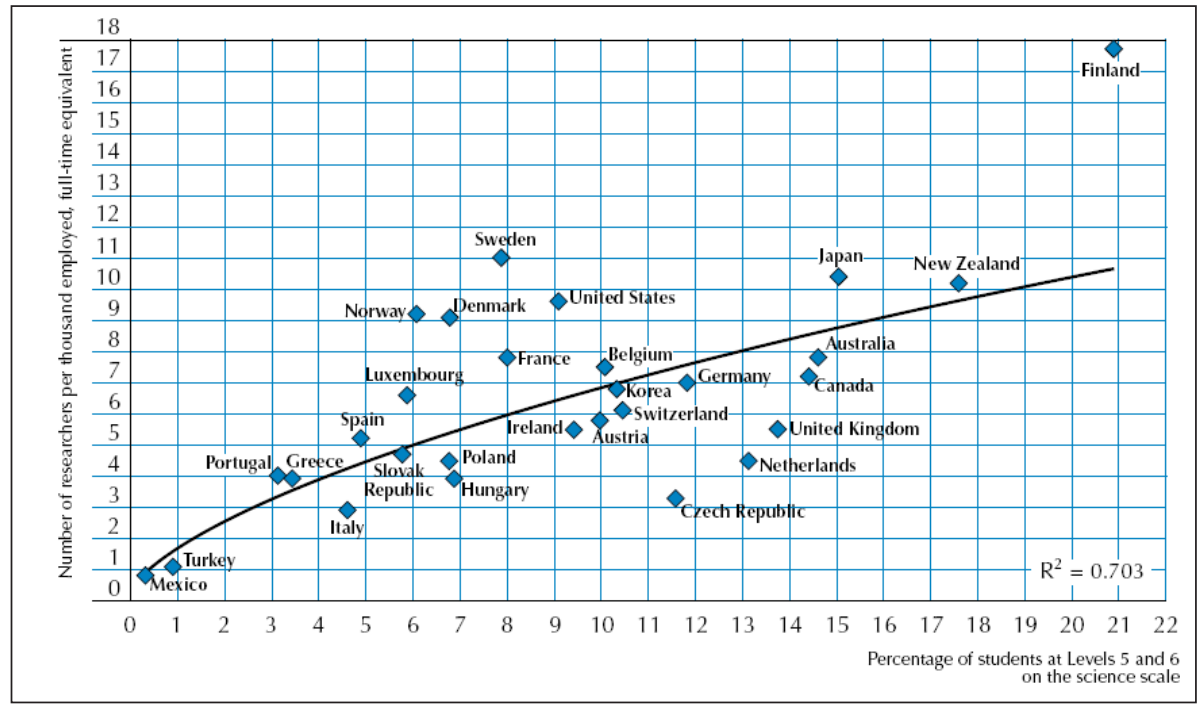

Fonte: TOP of the Class: high Performers in Science in PISA 2006.

Paris: OCDE, 2009. Tabla 2.1a.

\section{Perspectiva general sobre los mejores de la clase}

Resumimos los resultados por áreas, género, origen socioeconómico, centro escolar, experiencia, actitudes y motivación hacia las ciencias.

1) Las áreas - En el Gráfico 5 se puede observar la distribución de la reserva general de talento con respecto a las tres áreas evaluadas y el solapamiento que se produce entre 
ellas. Respecto al solapamiento conviene aclarar que los puntos de corte de cada una de ellas no coinciden pues la naturaleza y contenido de cada una de las áreas hace que dichos puntos de corte sean diferentes. Así, el punto de corte para Top Performers en lectura (medida en una escala de 1 a 5 ) es el nivel 5 que corresponde a una puntuación superior a 625,61; en matemáticas los Top Performers corresponden a los niveles 5 y 6 en una escala de 1 a 6 e incluye las puntuaciones superiores a 606,99.

Gráfico 5 - Media del solapamiento en lectura, ciencias y matemáticas de los alumnos Top Performers

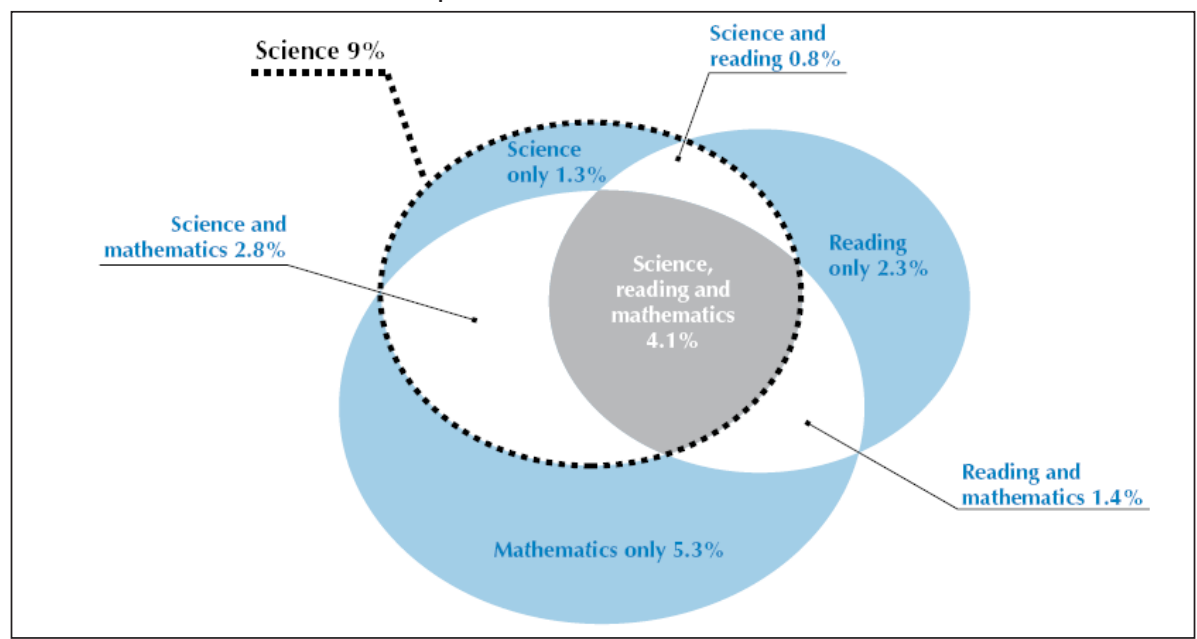

Fonte: TOP of the Class: high Performers in Science in PISA 2006.

Paris: OCDE, 2009. Tabla A2.1a.

El Gráfico 5 muestra que el 17,9 \% son Top Performers en alguna de las tres áreas y el $4,1 \%$ de los alumnos estudiados son excelentes en las tres áreas al mismo tiempo o en lectura, matemáticas y ciencias (El 82,1\% no tiene alto rendimiento en ninguna de las tres áreas evaluadas). El porcentaje más alto de Top Performers corresponde a matemáticas (5,3\%), seguido de lectura $(2,3 \%)$ y ciencias $(1,3 \%)$; el más bajo a los Top Performers en ciencias y lectura $(0,8 \%)$. También es importante resaltar que el $44 \%$ de los alumnos con rendimiento excelente o Top Performers en ciencias (9\%) son también Top Performers en matemáticas o en lectura o en matemáticas y lectura.

2) El género - Otra perspectiva general del estudio es su distribución por género. Considerando las tres áreas juntas, no hay diferencias significativas en cuanto al número de chicos y chicas Top Performers en los paises de la OCDE. De media, son Top Performers en las tres áreas al mismo tiempo el 4,1\% de las chicas y 3,9\% de los chicos y lo son en al menos una de las áreas el 18,6\% de las chicas y el 17,3\% de la muestra de chicos (Gráfico 6). 
Gráfico 6 - Datos globales de Top Performers y diferenciación por género de los estudiantes que destacan en las tres áreas en su conjunto

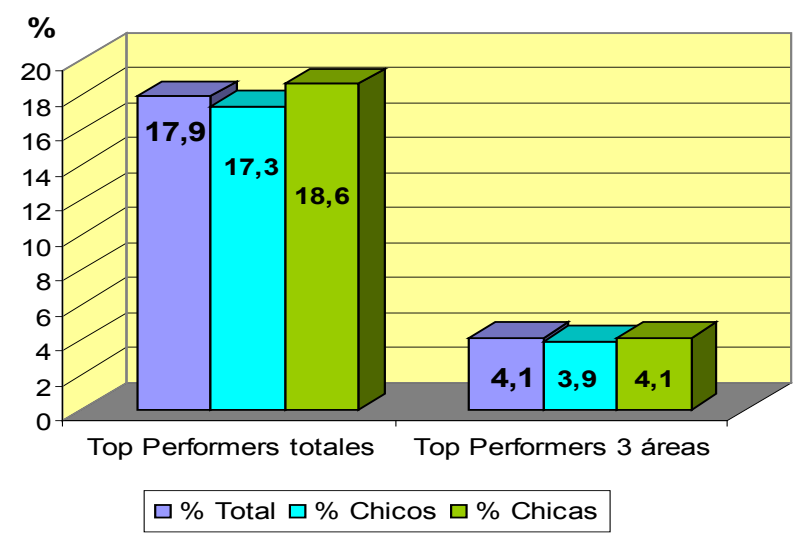

Fonte: TOP of the Class: high Performers in Science in PISA 2006. Paris: OCDE, 2009. Tabla A2.1a.

Esto promedios esconden una variación significativa entre países así como diferencias de género también significativas con respecto a las áreas. Mientras que la distancia entre los que son Top Performers solo en ciencias es muy corta: 1,1\% chicas y $1,5 \%$ chicos; la distancia es más amplia en lectura: $3,7 \%$ chicas y $0,8 \%$ chicos; y en matemáticas: $3,7 \%$ chicas y 6,8\% chicos (Gráfico 7).

Gráfico 7 - Diferencias de género de Top Performers solo en un área

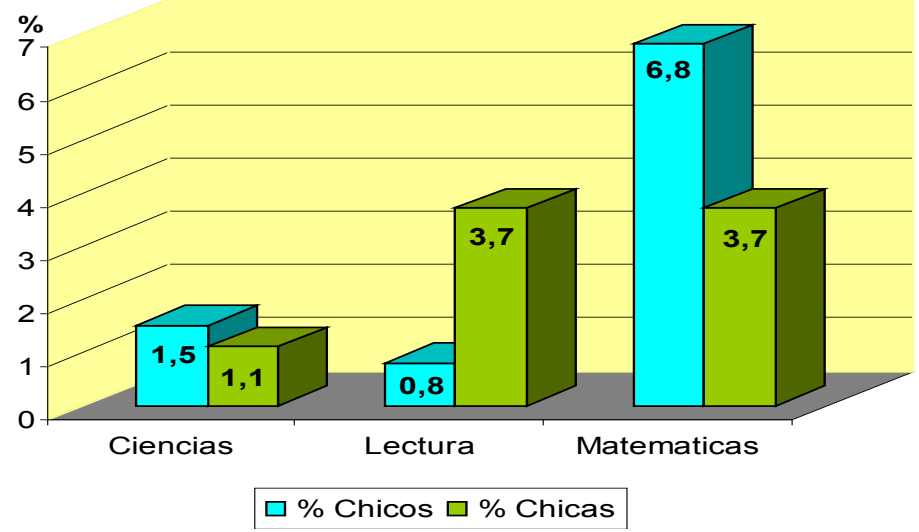

Fonte: TOP of the Class: high Performers in Science in PISA 2006.

Paris: OCDE, 2009. Tabla A2.1b. 
3) El origen socio-económico - En un país típico de la OCDE alrededor de un cuarto de los Top Performers en ciencias procede de un entorno socio-económico por debajo de la media del pais. En general son alumnos no inmigrantes que hablan en su hogar la lengua en que se realiza el test, pero en algunos países los inmigrantes y las minorías lingüisticas también alcanzan la excelencia en ciencias como ocurre con Holanda y Eslovenia.

4) El centro escolar - Suelen asistir a centros con población escolar que se caracteriza por su alto rendimiento y proceden de un entorno socio-cultural favorable. Han analizado las siguientes características del centro escolar:

- Tipo de centro - Una cuestión que requiere análisis es la distribución del alumnado Top Performers en los centros educativos. Es decir, si asisten a centros especiales "dedicados" a ellos o si bien asisten a centros donde no se concentran. El Gráfico 8 muestra, sobre fondo azul, los porcentajes de Top Performers que asisten a centros donde no hay alumnos top:

Gráfico 8 - Porcentaje de estudiantes que asisten a centros donde no hay Top Performers ${ }^{3}$

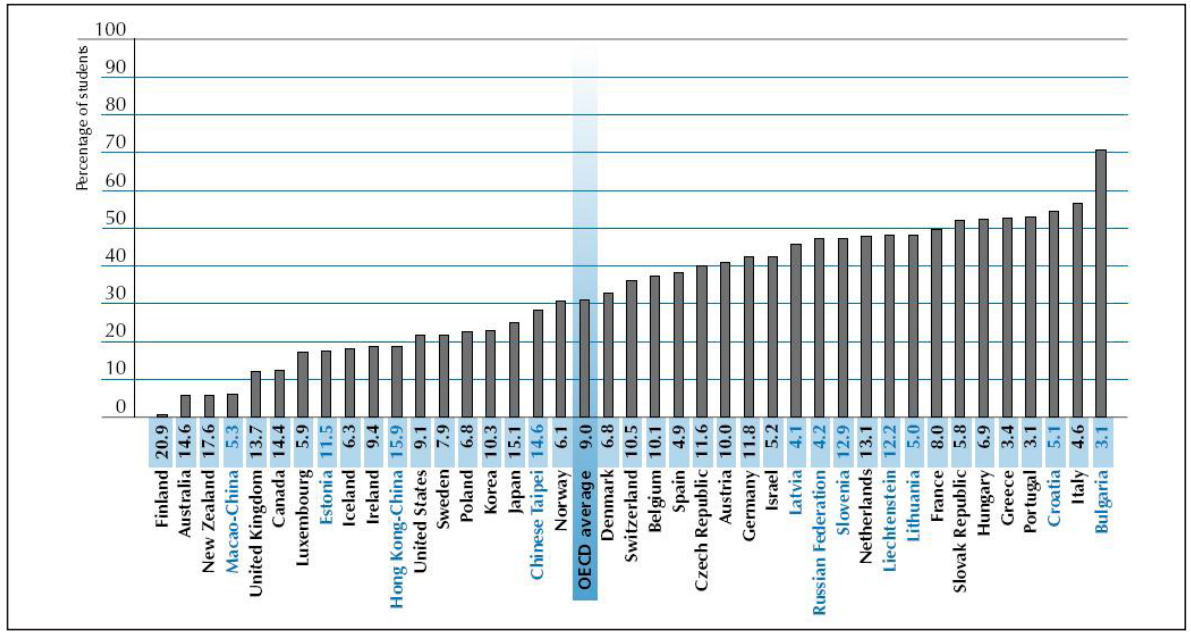

Fonte: TOP of the Class: high Performers in Science in PISA 2006.

Paris: OCDE, 2009. Tabla A2.6.

En paises como Finlandia, Australia y Nueva Zelanda, más del 90\% de los alumnos están en centros donde asisten Top Performers. En España, con un 4,9\% de Top

3 A la hora de analizar los datos hay que tener en cuenta que el porcentaje de Top Performers del gráfico se refiere a Ciencias, que incluye tanto los que sobresalen solo en ciencias como los que lo hacen en ciencias más otra disciplina, es decir, quedan excluidas las otras áreas cuando no se dan con Ciencias. Además de lo anterior se debe tener en cuenta que los porcentajes que se muestran de Top Performers provienen de sumar los porcentajes de alumnos en los niveles 5 y 6 de la escala de Ciencias. 
Performers en Ciencias (4,6\% en el nivel 5 y $0.3 \%$ en el nivel 6$)$, un porcentaje superior al 60\% asiste a centros donde hay Top Performers. Sin embargo, si observamos los porcentajes de Top Performers en Bélgica y la República Checa, aunque el porcentaje de estudiantes que asiste a centros con Top Performers es similar al de España, su porcentaje de Top Performers es muy superior, 10,1\% y 11,6\% respectivamente. Es decir, otras variables inciden en las diferencias de rendimiento que se observan entre estos países.

PISA encuentra que la diferencia entre el rendimiento medio de los centros varía considerablemente dependiendo del país. En los países de la OCDE la diferencia media en las puntuaciones en ciencias entre los centros a los que asisten Top Performers y los centros donde asisten Lowest Performers era de unos 104 puntos (La desviación estándar del rendimiento medio en Ciencias es 100). Constata dos situaciones diferenciadas entre los paises: a) países con poca variación entre centro: El caso más claro es Finlandia donde la distancia entre los centros con Top Performers y Lowest Performers es pequeña (30 puntos). Otros países son Noruega, Suecia y Polonia, con una distancia entre 40 y 51 puntos entre los dos grupos de rendimiento extremo. El caso de Finlandia sobresale porque no sólo presenta un porcentaje elevado de estudiantes Top Performers (20,9\%) y Strong Performers (32,2\%), sino que es muy bajo el porcentaje de alumnos que están por debajo del nivel 1 y en el nivel 1 ( $0,5 \%$ y $3,6 \%$ respectivamente) lo que indica un sistema educativo con una enseñanza de gran calidad; b) países con gran variación entre centros: En Holanda, Alemania, Hungría, Francia y Austria, entre otros, la diferencia en rendimiento medio en ciencias entre los centros donde asisten Top Performers y Lowest Performers es hasta tres veces superior al de los paises mencionados en el anterior apartado, lo que supone una desviación estándar y media o unos 150 puntos. En estos paises, los estudiantes Top Performers se encuentran en centros en los que el alumno medio suele rendir bien.

- Entorno socioeconómico de los centros - En el Gráfico 9 se puede ver la relación entre las diferencias socioeconómicas y de rendimiento entre centros con Top y Strong performers. En los paises donde las diferencias socioeconómicas son más grandes es donde las diferencias en rendimiento también son más grandes. Los estudiantes Top Performers asisten, fundamentalmente, a centros donde el alumnado en su conjunto procede de un entorno socioeconómico aventajado. En los países de la OCDE la diferencia media entre los centros a los que asisten Top Performers y Strong Performers es 0,15 puntos del índice socioeconómico. Esta diferencia es menor en paises con alta proporción de Top Performers. El contexto socioeconómico del centro está más fuertemente relacionado con los resultados de los alumnos que el estatus socioeconómico individual. 
Gráfico 9 - Relación entre diferencias socioeconómicas y de rendimiento entre centros con Top y Strongperformers

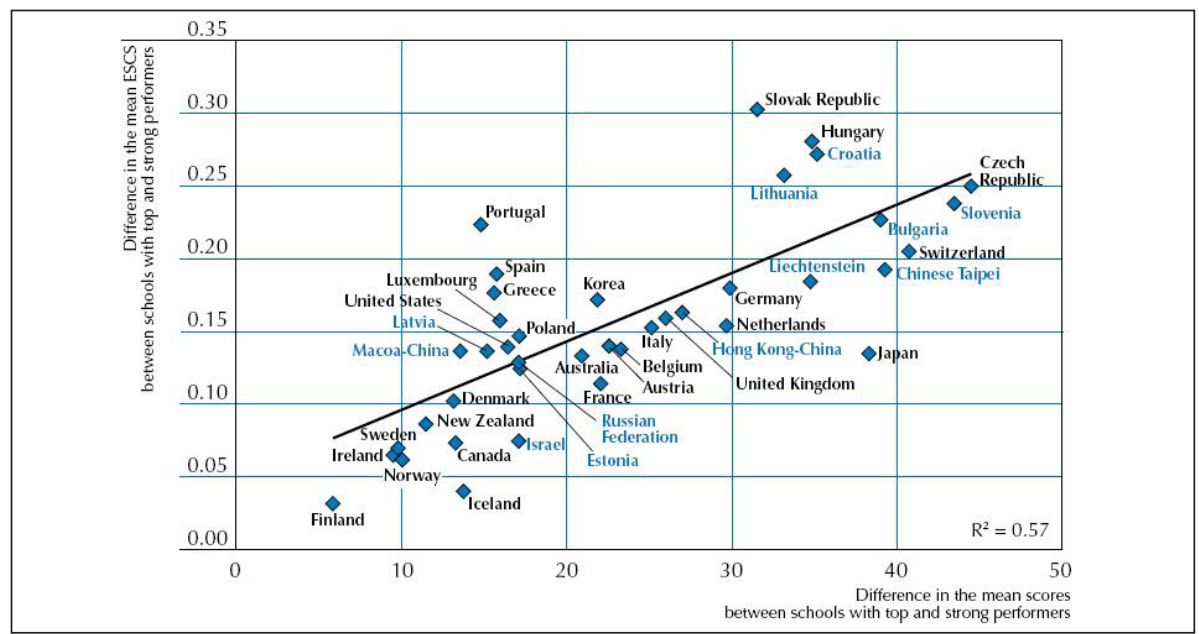

Fonte: TOP of the Class: high Performers in Science in PISA 2006.

Paris: OCDE, 2009. Tabla A2.6b.

- Centros públicos y centros privados - Define como centros públicos a aquellos que son gestionados por una autoridad pública, una agencia gubernamental o un consejo directivo autorizado o elegido de forma pública. Partiendo de esta base, el porcentaje medio de Top Performers en los países de la OCDE es el $9 \%$ en los centros públicos y el $14 \%$ en los centros privados. Importantes excepciones son Japón, Luxemburgo y la economía social China Taipei, que muestran más altas proporciones de Top Performers en centros públicos.

- Selección de estudiantes - Considera los siguientes aspectos: a) admisión y agrupamiento de los alumnos; b) expediente académico. Las políticas de admisión y agrupamiento establecen marcos dirigidos a la selección de los estudiantes para programas educativos o para su ubicación según metas profesionales. Hay paises donde se dan grandes diferencias de rendimiento dependiendo del programa y del centro, o donde la segregación socioeconómica es muy rígida. Parece que los centros más efectivos tienen más éxito a la hora de atraer a los alumnos más motivados y a los mejores profesionales de la enseñanza, produciéndose una fuga de cerebros de estudiantes y profesorado hacia ellos. En general, los centros donde el expediente académico es un prerrequisito para la admisión del alumno tienen más Top Performers que los centros donde no se sigue este criterio (Gráfico 10). 
Gráfico 10 - Selección de alumnado según el expediente académico

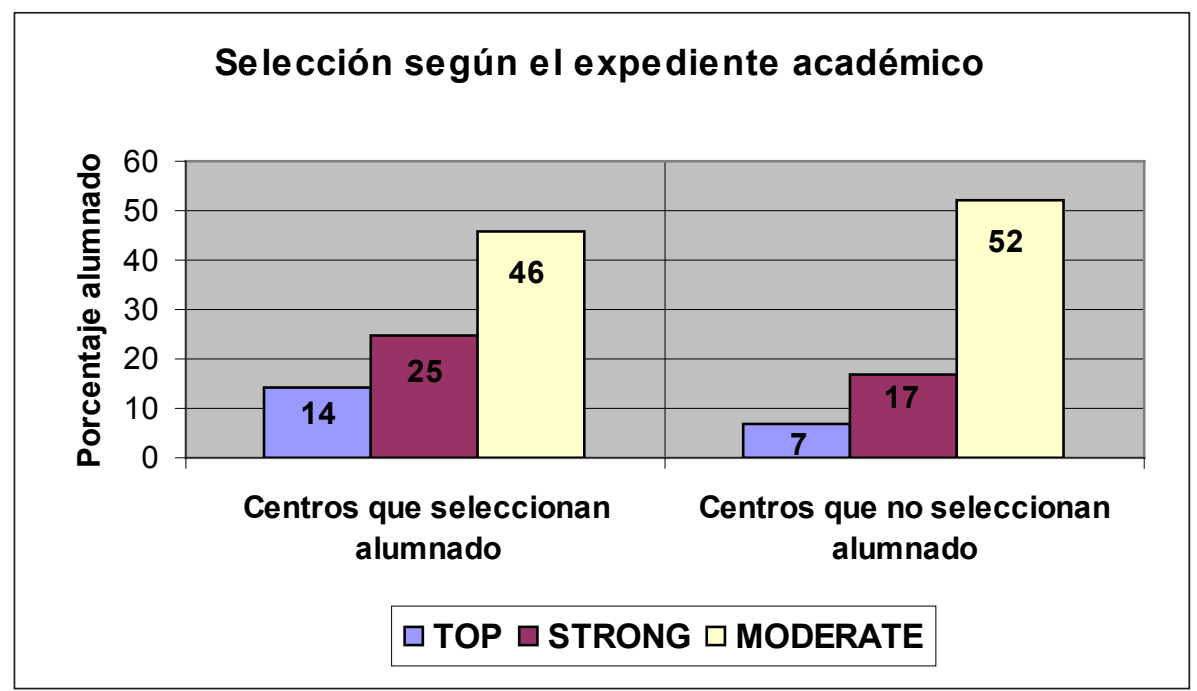

Fonte: TOP of the Class: high Performers in Science in PISA 2006. Paris: OCDE, 2009.

Estas diferencias a nivel de centros no tienen incidencia a nivel del sistema educativo. Es decir, los países con una proporción más alta de estudiantes en centros selectivos no alcanzan resultados generales globales mejores. Teniendo en cuenta lo anterior, podría pensarse que una parte del alumnado con talento, la de aquellos que no pueden elegir, se encuentra distribuido en otros centros donde probablemente no reciben la atención que sería deseable y, por este motivo, su rendimiento se encuentra por debajo de su capacidad.

5 - Experiencia, actitudes y motivaciones para el rendimiento excelente. Analizan tres grandes dimensiones: experiencia o tiempo medio dedicado a la enseñanza y el aprendizaje de las ciencias; actitudes o percepción de las clases de ciencias y actividades relacionadas con las ciencias y motivación o características del estudiante Top Perfomer en general y del estudiante Top Perfomer relativamente desmotivado.

- Experiencia - Consideran dos aspectos: a) horas de dedicación a las ciencias en el centro; b) clases de Ciencias fuera del centro escolar. Los estudiantes Top Performers reciben de media más tiempo de instrucción en ciencias que cualquiera de los otros grupos, reciben 4 horas a la semana. Los Strong Performers reciben 3,5 horas semanales y 2 horas los Low Performers. Señala que si entre los alumnos de 15 años se dan notables diferencias en el tiempo de exposición a las ciencias, éste factor puede ser una posible explicación para las diferencias de rendimiento, diferencias que serán más acusadas en edades posteriores cuando las ciencias se convierten en una materia que elige 
el alumno. Por otra parte, la instrucción fuera de la escuela suele perseguir profundizar en el conocimiento del alumno o elevar su bajo rendimiento. De media en los paises de la OCDE, los Low Performers reciben 45 minutos de instrucción extraescolar, mientras que los Top Performers reciben media hora o menos. Esta diferencia de 15 minutos a la semana no compensa las dos horas adicionales que reciben los Top Performers en la escuela.

- Actitudes - Consideran dos aspectos: a) percepción de las clases. b) actividades relacionadas con las ciencias. Plantea si hay diferencias en el rendimiento dependiendo del enfoque metodológico y los resultados en ciencias. A través de un cuestionario al alumnado recabó información que permitía la construcción de cuatro índices relacionados con cuatro estrategias de enseñanza: 1) estrategias basadas en modelos o aplicaciones de la ciencia, 2) investigaciones científicas, 3) participación activa y 4) debates entre los estudiantes sobre sus ideas y comprensión. Los resultados no son fácilmente interpretables y sugieren que cada enfoque podria ser mejor con determinado tipo de alumnos (top, strong, lowest perfomers). Asimismo elaboraron un índice de actividades relacionadas con las ciencias que trata de sacar a la luz en qué medida influye en el rendimiento el hecho de que los estudiantes se sientan atraídos por actividades relacionadas con esa materia vía programas de televisión, lectura de libros sobre temas científicos, programas de radio, revistas, artículos de periódicos 0 asistencia a clubs de ciencias. Se observa una relación alta y directa entre el rendimiento en ciencias y la frecuencia de participación en actividades relacionadas con las ciencias en cada uno de los países de la OCDE. De forma significativa en la mayoría de los países los Top Performers informaban de realizar más actividades científicas que los Strong Performers y que el resto de los grupos.

Puesto que algunas de estas actividades tienen lugar fuera del centro escolar, es lógico pensar que estén relacionadas con el estatus socioeconómico. Para ver hasta qué punto se da esta relación se hizo un ajuste del índice socioeconómico y se encontró que en todos los paises de los que se tienen datos, excepto la economía asociada Macao-China, la diferencia entre Top Performers y Strong Performers seguia siendo estadisticamente significativa. En promedio los Top Performers seguian estando un cuarto de desviación estándar por encima de los Strong.

Como parte de la evaluación también se pasó un cuestionario a padres en 16 paises, relativo a la realización de este tipo de actividades por parte de sus hijos. En 10 de los 16 países y economías los padres de los Top Performers informaron de que sus hijos habian hecho este tipo de actividades con más frecuencia que los de los Strong Performers.

- Motivación - Consideran dos grupos analizando varios aspectos dentro de ellos: a) características del estudiante Top Perfomer;b) características del 
estudiante Top Perfomer relativamente desmotivado. Respecto del primero, los Top Perfomer tienen mayor interés general por las ciencias (Quadro 1) que el grupo de los Strong Perfomer; se divierten más durante el aprendizaje de las ciencias (Quadro 2), tienen una mayor motivación instrumental para aprenderlas pues, en promedio, la mayoría de los estudiantes Top Performers informan de que estudian ciencias porque lo consideran útil (81\%), porque mejorará sus perspectivas profesionales $(76 \%)$ o porque piensan que lo necesitarán en sus futuros estudios (70\%), dándose marcadas diferencias entre este grupo y los demás grupos de rendimiento, incluida la comparación entre Top y Strong, siendo significativas las diferencias en todos los paises de la OCDE excepto en Grecia y Portugal, otorgan mayor importancia que los demás grupos a rendir bien en las distintas materias, tienen más confianza en sí mismos que el resto de los grupos y, a tal efecto, PISA ha elaborado un indice de autoeficacia en ciencias, definido como el nivel de confianza para afrontar tareas científicas específicas y muestran que los Top tienen un autoconcepto muy sólido en todos los paises estudiados y que el $70 \%$ de ellos responden que le resultaban fáciles las cuestiones relacionadas con las ciencias descendiendo el porcentaje al 55\% en el caso de los Strong Perfomers. Asimismo muestra que el $87 \%$ de los estudiantes consideran importantes las ciencias pero el $57 \%$ no las relacionan con su vida, extremo este último que cambia en el caso de los top (Quadro 3). Finalmente los Top, respecto de los Strong, destacan en su mayor interés por trabajar en una profesión relacionada con las ciencias y, en promedio, entre el 55\% y el 59\% se sentía informado sobre dónde encontrar información general sobre el tema descendiendo el porcentaje si se concretaba en aspectos como empresarios y empresas científicas.

Quadro 1 - Interés en temas relacionados con las ciencias

\begin{tabular}{|l|l|l|}
\hline Interés en temas de ciencias & StrongPerformers & Top Performers \\
\hline Biología humana & $74 \%$ & $77 \%$ \\
\hline Química & $59 \%$ & $72 \%$ \\
\hline Física & $57 \%$ & $69 \%$ \\
\hline Astronomía & $62 \%$ & $67 \%$ \\
\hline Diseño de experimentos & $50 \%$ & $58 \%$ \\
\hline Biología de las plantas & $51 \%$ & $56 \%$ \\
\hline Geología & $47 \%$ & $52 \%$ \\
\hline Requisitos para la explicación cientifica & $41 \%$ & $51 \%$ \\
\hline
\end{tabular}

Fonte: TOP of the Class: high Performers in Science in PISA 2006. Paris: OCDE, 2009. Tabla 3. 
Quadro 2 - Diferencias entre Strong y Top Performers en la gratificación del aprendizaje de ciencias

\begin{tabular}{|l|l|l|}
\hline $\begin{array}{l}\text { Las ciencias como aprendizaje } \\
\text { gratificante }\end{array}$ & Strong Performers & Top Performers \\
\hline $\begin{array}{l}\text { Disfruto adquiriend o nuevo } \\
\text { conocimiento en ciencias }\end{array}$ & $78 \%$ & $87 \%$ \\
\hline Me interesa aprender ciencias & $73 \%$ & $85 \%$ \\
\hline $\begin{array}{l}\text { Generalmente lo paso bien aprendiendo } \\
\text { temas de ciencia }\end{array}$ & $72 \%$ & $83 \%$ \\
\hline Me gusta leer sobre ciencia & $60 \%$ & $75 \%$ \\
\hline $\begin{array}{l}\text { Me siento bien haciendo problemas } \\
\text { de ciencia }\end{array}$ & $53 \%$ & $68 \%$ \\
\hline
\end{tabular}

Fonte: TOP of the Class: high Performers in Science in PISA 2006.

Paris: OCDE, 2009. Tabla 3.1.

Quadro 3 - Contraste Top Strong Performers. Valor personal de las ciencias

\begin{tabular}{|l|l|l|}
\hline Valor Personal de las Ciencias & Strong Performers & Top Performers \\
\hline $\begin{array}{l}\text { La ciencia me ayuda a entender las cosas } \\
\text { que me rodean. }\end{array}$ & $82 \%$ & $89 \%$ \\
\hline $\begin{array}{l}\text { Utilizaré las ciencias de muchas formas } \\
\text { cuando sea adulto }\end{array}$ & $70 \%$ & $80 \%$ \\
\hline La ciencia es relevante para mí & $64 \%$ & $76 \%$ \\
\hline $\begin{array}{l}\text { Cuando acabe la escuela tendré muchas } \\
\text { oportunidades para usar las ciencias. }\end{array}$ & $65 \%$ & $76 \%$ \\
\hline $\begin{array}{l}\text { Algunos conceptos de la ciencia me ayudan a } \\
\text { comprender mi relación con otras personas. }\end{array}$ & $58 \%$ & $60 \%$ \\
\hline
\end{tabular}

Fonte: TOP of the Class: high Performers in Science in PISA 2006.

Paris: OCDE, 2009. Tabla 3.4.

Respecto de los Top Perfomers en ciencias relativamente desmotivados, PISA los define en PISA como aquellos alumnos que informaron tener niveles de motivación inferiores a la motivación media en ciencias mostrada por los Strong Performers en el indice de motivación para un futuro orientado a las ciencias. Esto sucede en el 34\% de Top Performers en los países de la OCDE, observándose diferencias significativas 
entre países. En un extremo estarían la República Eslovaca, Latvia y Bulgaria, donde alrededor de la mitad de Top Performers informan de falta de motivación. En el otro extremo se encuentran Francia, Finlandia y Holanda en los que menos de un cuarto de Top Performers informan de estos niveles. En España el porcentaje de estos alumnos está cerca del $30 \%$ y se sitúa en el sexto lugar e después de Francia, Finlandia, Holanda, Japón y República Checa.

Dado el alto porcentaje de alumnos Top Performers en ciencias relativamente desmotivados, saber quiénes son y qué les caracteriza puede suponer información altamente relevante para la elaboración de políticas educativas. Se analizaron 19 paises en los que había suficientes datos: Hong-Kong China, Estonia, Finlandia, República Eslovaca, Bélgica, Japón, Reino Unido, República Checa, China Taipei, Nueva Zelanda, Australia, Corea, Canadá, Holanda, Alemania, Estados Unidos, Eslovenia, Liechtenstein y Austria y se puede concluir lo siguiente:

- Comparados con los Top Performers motivados, no pertenecen necesariamente a un entorno socioeconómico desfavorecido si se les;

- Se observan pocas diferencias de género entre los Top Performers motivados y los relativamente motivados. En 11 países no se dan diferencias de género aunque las chicas tienen más probabilidad de serlo en Bélgica, Holanda, Corea, Japón y el Reino Unido, así como en las economías asociadas China Taipei y Hong Kong-China. Sólo en la República Checa los chicos tienen más probabilidad que las chicas de ser Top Performers relativamente desmotivados;

- La diferencia entre motivados y menos motivados es mayor cuando se analiza la experiencia de aprendizaje de ciencias. Los motivados pasaban más tiempo en clases de ciencias en la escuela en 13 paises, aunque no es posible inferir cual es la causa y el efecto pues una posible explicación es que los motivados pasan más tiempo en ciencias porque sienten más motivación;

- La diferencia es más significativa cuando se valora el aprendizaje de ciencias como una actividad gratificante. En los 19 paises analizados los estudiantes motivados disfrutaban más aprendiendo ciencias que los relativamente desmotivados. La diferencia entre ambos grupos es al menos tres cuartos de desviación estándar en el índice de diversión con las ciencias, y en algunos países esta diferencia alcanza una desviación estándar o más;

- Los Top Performers tienden a realizar con más frecuencia en su vida diaria actividades relacionadas con las ciencias través de programas radio y televisión, páginas web, libros, revistas o artículos en periódicos sobre ciencias, asistir a club de ciencias. La diferencia en el índice de actividades relacionadas con las ciencias entre los motivados y menos motivados oscila desde media desviación estándar hasta tres cuartos de desviación estándar;

- Respecto a la información que les dan en el centro, los motivados informan con más frecuencia que los menos motivados recibir suficiente información, destrezas básicas y conocimiento para realizar una carrera de ciencias. La di- 
ferencia entre los dos grupos en el índice de información al estudiante sobre carreras relacionadas con las ciencias oscila entre un cuarto de desviación estándar en Bélgica y la economía asociada Hong Kong-China a dos tercios de desviación estándar en Holanda, Lichtenstein y República Checa.

\section{Alumnos con rendimiento excelente}

Resumimos los resultados obtenidos en los estudios realizados sobre dos grupos de alumnos con rendimiento excelente en la Comunidad de Madrid, alumnos que fueron elegidos por haber obtenido Premio Extraordinario de Bachillerato, en un caso, y en el otro por haber obtenido Beca de Excelencia para estudiar en alguna de las universidades madrileñas. Las cuestiones a las que se pretenden dar respuesta son: ¿Qué capacidades tienen chicos y chicas con alto rendimiento, cómo las utilizan, existen diferencias entre ambos grupos? ¿Cómo influye la escuela y la familia en el desarrollo del alto rendimiento?

\section{Alumnos con Premio Extraordinario de Bachillerato}

La tabla 3 recoge la muestra estudiada en dos momentos temporales o en los cursos 2000-01 y 2003-04, respectivamente, los padres y profesores de dichos alumnos que accedieron a colaborar y los instrumentos de recogida de datos empleados en cada caso.

Tabla 3 - Muestra e instrumentos utilizados en el seguimiento de los alumnos PEB

\begin{tabular}{|c|c|c|}
\hline ESTUDIO 2000-01 & $\begin{array}{l}\text { MUESTRA } \\
\text { Varones Mujeres Total }\end{array}$ & INSTRUMENTOS \\
\hline Alumnos PEB & 172138 & $\begin{array}{l}\text { Cuestionario ad hoc/ Tests/ } \\
\text { Grupos de discusión libre de } \\
\text { ambos sexos }\end{array}$ \\
\hline Padres de los alumnos PEB & 212849 & $\begin{array}{l}\text { Cuestionario ad hoc/Grupos de } \\
\text { discusión libre de ambos sexos }\end{array}$ \\
\hline Profesores de los alumnos PEB & 8614 & \\
\hline \multicolumn{3}{|l|}{ ESTUDIO 2003-04 } \\
\hline Alumnos PEB & 171431 & $\begin{array}{l}\text { Cuestionarios ad hoc/ Tests/ } \\
\text { Rendimiento académico/ } \\
\text { Grupos de discusión libre sólo } \\
\text { de chicas, sólo de chicos y de } \\
\text { ambos sexos }\end{array}$ \\
\hline
\end{tabular}

Fonte: Jiménez; Álvarez; Gil; Murga; Téllez (2006).

Para optar a dicho premio es preciso tener una media de sobresaliente en los estudios de bachillerato y superar una prueba compleja. Los que finalmente lo obtienen, pueden optar al Premio Nacional de Bachillerato. Se otorga un Premio Extraordinario de 
Bachillerato (PEB) por cada mil alumnos matriculados en el último curso de bachillerato objeto de la convocatoria anual de dichos premios.

1) Características sociológicas de los alumnos - son alumnos cuyas edades oscilaban entre los 18 (53\%) y 19 años (47\%) en el momento de recoger los datos predominando ligeramente las chicas (53\%). Tienen dos (50\%) o tres hermanos (24\%), son primogénitos (63\%) u ocupan el segundo lugar en la fratría (18\%). La mitad de los padres tienen 50 o menos años y la mitad de las madres 48 o menos años por lo que son hijos de madres maduras, añosas para su tiempo. Proceden de familias con escasa separación o divorcio (5\%) y con nivel cultural medio o alto pues el $44 \%$ de los padres y el $42 \%$ de las madres tienen estudios superiores, son hijos de padres (95\%) y madres (65\%) que trabajan fuera del hogar en trabajos bien o medianamente considerados. El 56\% estudió en centros públicos. En la universidad obtienen buen rendimiento, realizan el curso que les corresponde por su edad y han elegido mayoritariamente carreras duras y prestigiosas pues la mitad estudia una ingeniería superior. La otra mitad se distribuye entre ciencias, medicina, biología y económicas y empresariales (19\%) y el 31\% en estudios de humanidades (JIMÉNEZ; AGUADO; ÁLVAREZ; GIL; JIMÉNEZ, 2002).

2) Resultados en los tests de capacidad - en 2000-01 los resultados en dichos tests los sitúan en el cuartil superior o próximo a él. En el Tests de Matrices Progresivas de Raven obtienen como grupo una puntuación centil normalizada de 78 con una desviación típica de 23. No hay diferencias significativas en función del género. Sí las hay en función de la opción de bachillerato elegida o ciencias, letras o estudios mixtos, a favor de los de ciencias, aunque no en todas las escalas. En el Test de Aptitudes Mentales Primarias obtienen puntuaciones centiles normalizadas algo superiores a la mediana en los factores verbal y espacial, inferior en el numérico (Pc. 44), y se sitúan en el cuartil superior en las pruebas de razonamiento y de fluidez verbal. No hay diferencias significativas en función del género. El eje letras, ciencias, mixto, sí arroja diferencias significativas en el subtest de razonamiento, a favor de los alumnos de ciencias.

En 2003-04 los resultados obtenidos confirman su capacidad. En el test WAIS oscilan entre el percentil 60 en la prueba "Búsqueda de símbolos" y el percentil 91 en la de "Información". Las diferencias de género no son estadísticamente significativas. En dicha prueba el $\mathrm{Cl}$ verbal es el más elevado $(132,62)$, seguido del $\mathrm{Cl}$ total $(128,77)$ y del $\mathrm{Cl}$ manipulativo (116,92), una buena puntuación en todos los casos. Las diferencias de género no son significativas en ninguno de los tres cocientes (JIMÉNEZ; ÁLVAREZ; GIL; MURGA; TÉLLEZ, 2005).

3) Intereses profesionales y creatividad - en el test de Intereses y Preferencias Profesionales (IPP) los percentiles se mantienen en niveles discretos y con fuertes dispersiones, pues oscilan entre los valores de 13 y 35 , y ello en los diferentes tipos 
de escalas dando la impresión de que la prueba no parece (¿aún?) adecuada para estos alumnos. Hay algunas diferencias estadísticamente significativas en función del género, destacando los chicos en los campos científico, técnico y militar y las chicas en el literario y en el económico-empresarial. También aparecen algunas diferencias significativas en torno al eje ciencias, letras, estudios mixtos; el grupo mixto destaca en el campo científico-experimental y psicopedagógico y el de ciencias lo hace en el científico-técnico. En el Test de Abreación Para evaluar la Creatividad, puntúan ligeramente por encima de la media de la población general en siete de las diecisiete escalas de la prueba, y en torno a la media en las restantes; la dispersión tiende a ser mayor que la de la población general. No hay diferencias significativas en función del género ni del tipo de estudios elegidos en bachillerato.

4) Educación familiar - la educación recibida ha sido exigente con hijos e hijas (una forma de discriminación sexual apuntada en los grupos de discusión es exigir intelectualmente menos a la hija y tener hacia ella expectativas más bajas) y les ha dotado de disciplina, hábitos, valores e instrumentos cognitivos valiosos para un recorrido escolar de fondo. La familia, conservadora o liberal, ha desarrollado en su seno estilos educativos democráticos y coherentes, cuidando el clima familiar y las normas que rigen la convivencia. Desde la infancia los padres han respondido con sencillez a las preguntas de los hijos, han fomentado sus logros, han sembrado expectativas en los hijos y hacia los hijos, han dado importancia al esfuerzo y a la determinación personal y han valorado el buen rendimiento. Por ejemplo, el grupo PEB destaca en afición lectora, dedicar tiempo al estudio, otorgar un lugar prioritario a las obligaciones escolares, dominio de idiomas, iniciación a la informática y valorar la autonomía y responsabilidad personal. No han tenido profesores particulares ni han alternado estudio y trabajo, salvo excepción. Las prioridades, la concentración de esfuerzos, las expectativas y los propios recursos cognitivos desarrollados por el alumno, figuran entre los factores responsables del alto rendimiento (JIMÉNEZ FERNÁNDEZ, 2006).

5) La escuela y los estereotipos existentes - a través de los grupos de discusión se analizan las percepciones, actitudes y valores de los alumnos PEB hacia la situación escolar y social de los más capaces que sucintamente resumimos (JIMÉNEZ; ÁLVAREZ; GIL; MURGA; TÉLLEZ, 2006). Alumnos, padres y profesores coinciden al concluir de modo independiente que la escuela no atiende a estos alumnos sino que se limita a repetir, repetir y repetir, y que pese al avance producido hacia la igualdad, perviven estereotipos, prejuicios y actitudes que discriminan doblemente a la mujer capaz dada su condición de mujer y de mujer con capacidad. El avance hacia la igualdad ha sido mayor entre la población más joven y más ilustrada y en los ámbitos más cultos y abiertos como la escuela, la universidad y las ciudades, pero aún en ellos, persisten reductos machistas soterrados bajo lo "políticamente correcto" y aparecen nuevos estereotipos o viejos estereotipos con nuevos formatos. En la escuela se refleja con contradicciones y luces 
propias. Los profesores son conscientes de que no se atiende a los más capaces porque otro tipo de alumnos tiene necesidades socialmente más perentorias. Sí perciben su valía y las sutiles diferencias que se dan hacia y entre los y las más capaces, a favor de aquellos.

Padres, profesores y alumnos coinciden al señalar que la capacidad cognitiva y el rendimiento escolar excelente se perciben como valores positivos en los chicos y en cierto modo también en las chicas, pero pervive con fuerza aquello de "a las muy listas no las quiere nadie"; las chicas muy brillantes despiertan cierto rechazo social entre sus iguales. Asimismo afloran algunos estereotipos diferenciados en la conducta social. Los chicos serian más nobles, simples y directos; las chicas más "enrevesadas y brujas". En la persistencia de estereotipos y en la aparición de otros estaría influyendo el propio comportamiento femenino, pues hay chicas que se resisten a afrontar el hecho de la igualdad, ya por el peso de los estereotipos y de la presión social, ya porque deben invertir mucha energía "inútil" en el intento, ya porque temen afrontar en soledad las consecuencias. El peso de ciertos estereotipos se mantiene hasta en la universidad pues hay profesores que encuentran difícil otorgar la máxima calificación a una chica aunque su rendimiento sea superior; el mejor debe seguir siendo un varón, estereotipo con muchas caras.

\section{Alumnos con Beca de Excelencia}

En este estudio los datos se recogieron en el curso 2004-05, la muestra está formada por 40 varones y 78 mujeres elegidas por disfrutar de una Beca de Excelencia. Las bases que regulan la convocatoria pública de dichas becas se contienen en la Orden 2000/2004, de 28 de mayo, del Consejero de Educación, por la que se convocan ayudas a alumnos con aprovechamiento académico excelente, para cursar estudios presenciales en las Universidades de la Comunidad de Madrid. Curso 2004-05 (B.0.C.M. núm. 131, jueves 3 de junio de 2004). Dichas becas pretenden atraer a los estudiantes que han obtenido rendimiento excelente en bachillerato y saben mantenerlo en la universidad, y promover la movilidad hacia Europa y América de los estudiantes universitarios capaces de mantener un rendimiento excelente en las universidades participantes en el programa IDEUM 2010 (Investigación y Docencia de Excelencia Universitaria Madrileña). La nota media necesaria para solicitar una beca en el caso de los alumnos de nuevo ingreso en la universidad era de 9,31 puntos. La distribución por carrera y género de la muestra estudiada se recoge en la Tabla 4. Nos ceñimos a dos ámbitos, el académico y las capacidades personales, analizados desde datos cuantitativos 0 resultados en las pruebas aplicadas, y cualitativos o reflexiones de los propios alumnos en los grupos de discusión. Se indaga en el principio de que el contexto incide directa e indirectamente en el desarrollo del alto rendimiento y de la alta capacidad. Para conocer más datos remitimos a una publicación anterior (JIMÉNEZ; MURGA; GIL; TÉLLEZ; TRILLO, 2010). 
Tabla 4 - Distribución de la muestra según carrera y género

\begin{tabular}{|c|c|c|c|c|}
\hline \multicolumn{2}{|l|}{ Sexo } & Varón & Mujer & Total \\
\hline \multirow{5}{*}{ Carrera } & Humanidades & $35,0 \%$ & $65,0 \%$ & $100,0 \%$ \\
\hline & CC. Experimentales & $12,4 \%$ & $86,5 \%$ & $100,0 \%$ \\
\hline & CC. de la Salud & $25,0 \%$ & $75,0 \%$ & $100,0 \%$ \\
\hline & CC. SS. JJ & $24,0 \%$ & $75,1 \%$ & $100,0 \%$ \\
\hline & Técnicas & $65,6 \%$ & $34,4 \%$ & $100,0 \%$ \\
\hline Total & & $34,0 \%$ & $66,0 \%$ & $100,0 \%$ \\
\hline
\end{tabular}

Fonte: Jiménez; Murga; Gil; Téllez; Trillo (2010, p. 134).

c) Capacidades - Las capacidades de chicos y chicas desde los resultados en el el test de Raven (Tabla 5) muestran claramente que el grupo obtiene unos valores superiores al grupo normal. Por ejemplo, su mediana se sitúa en el valor 85 , muy superior al 50 que sería el valor esperable en el grupo normal. La dispersión es pequeña con una amplitud intercuartílica de 32, lo que significa que entre los valores de 63 y 95 están el 50\% de los casos, una distribución centrada en los valores superiores. Los resultados obtenidos no muestran diferencias significativas en función del género.

Tabla 5 - Percentiles en el test RAVEN

\begin{tabular}{ll|l|l|l|l|l|l|l}
\hline & & \multicolumn{2}{l|}{ Percentiles } \\
\cline { 3 - 9 } & & 5 & 10 & 25 & 50 & 75 & 90 & 95 \\
\hline $\begin{array}{l}\text { Promedio } \\
\begin{array}{l}\text { ponderado } \\
\text { (definición 1) }\end{array}\end{array}$ & $\begin{array}{l}\text { Raven II } \\
\text { estandarizado }\end{array}$ & 29,55 & 45,00 & 62,75 & 85,00 & 95,00 & 97,00 & 97,00 \\
\hline Bisagras de Tukey & $\begin{array}{l}\text { Raven II } \\
\text { estandarizado }\end{array}$ & & & 63,00 & 85,00 & 95,00 & & \\
\hline
\end{tabular}

Fonte: Jiménez; Murga; Gil; Téllez; Trillo (2010, p. 135).

En el Test de Aptitudes Mentales Primarias (PMA), que mide los factores verbal, espacial, razonamiento abstracto, cálculo y fluidez verbal, en la Tabla 6 podemos comprobar las puntuaciones directas obtenidas por el grupo y las puntuaciones estandarizadas. Todos los valores normalizados son superiores a las puntuaciones directas, excepción hecha de los valores "mínimos" obtenidos en el subtest verbal y en cálculo numérico, en los que en coherencia con la superioridad general manifestada, los valores máximos y la media siguen siendo claramente superiores en el PMA estandarizado. La única diferencia significativa aparece en razonamiento abstracto y favorece a las mujeres. 
Tabla 6 - Estadisticos descriptivos de la prueba PMA

\begin{tabular}{l|l|l|l|l|l}
\hline & N & Mínimo & Máximo & Media & Desv. Típica \\
\hline PMA verbal & 117 & 20 & 49 & 32,32 & 6,283 \\
PMA verbal estandarizado & 117 & 5 & 98 & 43,15 & 23,787 \\
PMA espacial & 117 & -13 & 54 & 28,59 & 14,202 \\
PMA espacial estandarizado & 117 & 1 & 99 & 54,20 & 29,407 \\
PMA razonamiento & 117 & 9 & 30 & 21,98 & 4,826 \\
PMA razonamiento estandarizado & 117 & 5 & 99 & 66,75 & 28,903 \\
PMA cálculo numérico & 117 & -2 & 41 & 19,39 & 7,961 \\
PMA cálculo numérico estandarizado & 117 & 1 & 98 & 32,79 & 27,415 \\
PMA fluidez verbal & 117 & 25 & 75 & 53,24 & 11,287 \\
PMA fluidez verbal estandarizado & 117 & 2 & 99 & 60,68 & 27,912 \\
\hline
\end{tabular}

Fonte: Jiménez; Murga; Gil; Téllez; Trillo (2010, p. 136).

d)Estrategias y hábitos de trabajo intelectual. Del Cuestionario de procesos, orientaciones y concepciones del aprendizaje y enseñanza del alumno universitaride J.Vermunt, se concluye que, en general, el grupo muestra buenas estrategias y hábitos de trabajo intelectual como corresponde a un grupo con alto rendimiento, extremo que se aprecia tanto en los ítems que puntúan por encima del grupo normativo y que apuntan a autonomía y autoexigencia en el aprendizaje, cuanto en los que rechazan por significar dependencia externa y trabajo superficial.

La forma típica de trabajo que manifiestan ante el estudio es relacionar los nuevos contenidos de un tema con el conocimiento previo que tienen de ese tema; intentan generalizar lo aprendido y a menudo utilizan lo que aprende en las asignaturas en actividades diferentes de sus estudios e intentan interpretar acontecimientos o situaciones de la vida diaria con ayuda de los conocimientos adquiridos en los estudios. Para comprobar su aprendizaje, cuando han estudiado un texto, intentan formular las ideas principales con sus propias palabras e intentan expresar el contenido de un párrafo del mismo modo, es decir, con sus propias palabras. Prestan atención especial a aquellas partes del curso y de las asignaturas que tienen una utilidad práctica e intentan ver las conexiones entre los contenidos de diferentes capitulos de un libro. Finalmente, ante las dificultades en el estudio, intentan determinar cuál es la causa de cada dificultad y si no comprenden bien un texto, buscan otros libros sobre el mismo tema que puedan aclararles los elementos ausentes o confusos. Las chicas son más autoexigentes y piensan, en mayor medida, que deben comprobar de formas diversas que dominan lo que estudian. 
e) En los grupos de discusión - chicos y chicas consideran que, en general, tanto en la escolaridad obligatoria como en el instituto y en la universidad, han recibido un tratamiento equitativo. A pesar de ello sí encuentran en ocasiones un tratamiento discriminatorio hacia las chicas, procedente tanto de los profesores como de los compañeros y de la propia familia. Abundan en ejemplos en la línea de los alumnos $\mathrm{PEB}$, apreciándose mayor discernimiento en el análisis de los hechos (JIMÉNEZ; ÁLVAREZ; MURGA; GIL; TÉLLEZ; TRILLO, 2006).

\begin{abstract}
Chica: ...incluso entre mis profesores, que son bastantes equitativos por lo general, si bien llega el profesor de turno y no te pone un diez por la media y llega el chico y sí se lo pone. Hay algún profesor muy machista en mi universidad y en el colegio pasaba igual. A mí literalmente me dijo un profesor que la nota más alta se la llevaba un chico porque para eso era chico (Grupo 2).
\end{abstract}

Chicos y chicas consideran que la educación y la enseñanza no se ajusta adecuadamente a las diferencias individuales. Critican las técnicas de motivación y las estrategias de enseñanza empleadas pues, en ocasiones, limitan el pensamiento divergente y creativo. En la elección de estudios dicen que en su caso ha primado la libertad y los intereses personales de cada cual. Son conscientes de que existe cierta presión social hacia los chicos y chicas para que elijan o desestimen determinadas carrera; pero, a la vez, también son conscientes de que estas dinámicas sociales están cambiando. Se trata de favorecer un cambio de mentalidad social que normalice la elección de estudios en función de las capacidades e intereses, sin situar el género como categoría convencional discriminatoria. En cualquier caso, parece ser que son los chicos los que reciben mayores presiones por parte de la familia para que no se adentren por elecciones tradicionalmente femeninas, consideradas social y profesionalmente más débiles. En el caso de las chicas capaces se admite más que elijan estudios convencionalmente masculinos o femeninos. Señalan que la influencia, positiva y negativa, de los profesores se convierte en un elemento condicionante de la elección de estudios.

Chica: Yo con los profesores siempre he tenido buena relación $\mathrm{y}$ he sentido que me apoyaban pero tampoco de una manera muy incisiva. Es decir, si tenia cualquier problema estaban encantados de ayudarme, pero a la hora de llevar las asignaturas el peso recaía sobre mí fundamentalmente. A nivel de instituto y de colegio siempre se suele apoyar a la gente que suele ir mal y hay muchas ayudas encaminadas a ayudarles, como grupos especificos...En cambio, hacia las personas que van bien hay mucho menos apoyo para procurar estimularles y depende mucho más de lo que tú hagas (Grupo 4).

Chico: Mi colegio era laico. De una forma muy sutil era: chico listo con buenas notas: ingeniero; chica lista con buenas notas: 
sanitario. En segundo de bachillerato éramos una clase de 15 personas y era el único chico (Grupo 5).

Chicos y chicas valoran los entornos diversos y la diversidad. La universidad es considerada un espacio diverso, caracterizado por muestras de respeto a la libertad personal y a la diversidad social. Asimismo señalan que es necesario repensar la oferta educativa en relación a las personas que la universidad pretende formar y a la sociedad que se desea promover. Los utilitarismos reducen materias y especialidades con tradición cultural y cierta proyección social; la limitación del número de plazas condena a la sequia a determinadas opciones estudiantiles aunque sean buenos alumnos.

"Chica: Yo no puedo entender que quiten historia del arte con el patrimonio histórico que tenemos" (Grupo 6).

Dentro del avance hacia la igualdad, chicos y chicas consideran que desde la familia y la escuela se tiene una percepción diferenciada de las capacidades de ambos géneros, que discrimina al femenino. Frente al mismo resultado, padres y profesores consideran que en el caso de los chicos se debe a la capacidad y en el de las chicas al esfuerzo y la constancia, extremo que no se sostiene desde los resultados de los tests, ni desde el cuestionario, ni desde las valoraciones de los grupos de discusión ni desde el rendimiento anterior obtenido por ellos y ellas. Más bien consideran que chicos y chicas se caracterizan por tener diferentes capacidades e intereses, y que éstos han sido y se verán condicionados socioculturalmente a través de las expectativas sociales y de los papeles asignados estereotipadamente a cada uno de los géneros, aunque a su juicio estos temas están cambiando.

Chico: Yo, el mero hecho de entrar a diferenciar si una persona lista es hombre o mujer lo veo un poco absurdo. Es como clasificar si los rubios son más inteligentes que los morenos, no creo que por ser mujer vayan a ser más tontas o más listas (Grupo 1).

Chicos y chicas consideran que las chicas son más inteligentes si bien las capacidades relativas a pensamiento abstracto, matemático y espacial se siguen asociando a los chicos. Por el contrario, se relaciona con las chicas una mayor capacidad emocional e interpersonal. Asimismo consideran que chicos y chicas hacen un uso distinto de sus capacidades; ellas hacen un uso más estratégico de las mismas, son más ordenadas y se esfuerzan más, extremo convergente con los datos del cuestionario y matizado en los grupos de discusión respecto del estereotipo simplista de que salen adelanten porque se esfuerzan más que los chicos. Este estereotipo asoma al valorar al profesorado y también lo exponían los alumnos PEB. Pese a ello, admiten un avance en el reconocimiento de la igualdad.

Chica: Porque a las mujeres siempre se nos ha considerado que se nos da mejor el contacto personal, las interrelaciones [...] (Grupo 5). 
Chica: Sí, a lo mejor no tienen tanto en cuenta la manera en que dicen las cosas y eso puede ser malo. Hombre, yo no siempre mido lo que digo pero a mí me gusta que la persona con la que estás hablando te sonría [...] (Grupo 7).

Chico: Lo que sí que es cierto es que hay algunos tipos de inteligencia que están más desarrollados que otros. A lo mejor las chicas tienen más facilidad o conocimientos que los chicos en un tipo de actividad mientras que los chicos los pueden tener en otras (Grupo 8).

\section{A modo de conclusión}

Es arriesgado concluir sobre qué factores están detrás del rendimiento de los mejores alumnos si bien hay que intentarlo pues un esbozo de conclusiones es exigible a todo trabajo científico. En ambos tipos de estudios o evaluación de PISA y estudio de dos grupos de alumnos españoles con rendimiento excelente, se aprecian concomitancias y no descubren nada totalmente nuevo sino que afinan en datos y matices cara a explicar el logro del rendimiento excelente en la realidad actual. Con todas las cautelas y a sabiendas de que hablamos de asociación o relación entre las variables, esbozamos las siguientes.

Las evaluaciones de PISA muestran lo que viene siendo una obviedad en los estudios sobre rendimiento escolar, que hay un pequeño grupo de alumnos que obtienen un rendimiento excelente en las tres competencias evaluadas o en lectura, matemáticas y ciencias, bien tomadas independientemente o tomadas en su conjunto, y que en el caso de España ese pequeño grupo de alumnos es prácticamente inexistente 0 , al menos, varios puntos inferior al promedio obtenido por los paises de la OCDE. Sobre el 4\% de los alumnos estudiados por la OCDE (2009) son excelentes en lectura, matemáticas y ciencias al mismo tiempo y sobre el 18\% tiene rendimiento excelente en alguna de las tres áreas. El 4,1\% de las chicas y el 3,9\% de los chicos sobresalen en las tres áreas y sobresalen en al menos un área el 18,6\% de las alumnas y el 17,3\% de los alumnos.

Los alumnos con rendimiento excelente proceden en su mayor parte de entornos socioeconómicos favorecidos, si bien el origen familiar es menos determinante para el rendimiento excelente que el entorno social y educativo del centro pues sobre la cuarta parte de los mejores alumnos proceden de entornos socioeconómicos desfavorecidos. Por otra parte, en los centros con alto rendimiento predominan los alumnos de entorno sociocultural favorable $y$, a su vez, estos centros atraen en mayor medida a los mejores estudiantes. Los centros más selectivos tienen más alumnos con rendimiento elevado. Aunque existen variaciones entre paises, los mejores alumnos estudian en centros públicos (9\%) en menor medida que en centros privados (14\%). 
Una característica muy importante para la obtención de alto rendimiento es el tiempo que el currículo escolar dedica al estudio de las materias, característica que empalma con los estudios clásicos sobre tiempo real de aprendizaje y eficacia escolar (SMYTH, 1985). Al evaluar la competencia científica PISA ha observado que los alumnos con rendimiento excelente en ciencias asisten a centros que dedican cuatro horas semanales a la enseñanza de las ciencias. Los alumnos con buen rendimiento reciben media hora menos de clase semanal y los de bajo rendimiento reciben sólo dos horas semanales. Las clases extraescolares que pueden recibir los alumnos, cuando existen, suponen unos treinta minutos semanales más dedicados al estudio lo que no llega a compensar las cuatro horas de exposición sistemática al aprendizaje.

El rendimiento excelente se relaciona con actitudes positivas hacia la educación y hacia el buen rendimiento, con la motivación positiva hacia el estudio, con el interés hacia distintas materias y sus proyección social y profesional, con un buen autoconcepto en el alumno, con sus expectativas académicas y profesionales y con el conocimiento que tiene de la utilidad de las materias de estudio en su entorno social, entre otros. Al centrarse en el estudio de las ciencias, PISA constata que los mejores alumnos tienen actitudes más positivas hacia las clases de ciencias y hacia determinados métodos de enseñanza y aprendizaje de esta materia, que el resto de los alumnos. Por ejemplo, de media, la mayoria de los estudiantes Top Performers informan de que estudian ciencias porque lo consideran útil (81\%), porque mejorará sus perspectivas profesionales (76\%) o porque piensan que lo necesitarán en sus futuros estudios (70\%) y tienen más información sobre estas cuestiones que los alumnos strong. La experiencia, la dedicación y la actitud de los alumnos contribuyen de forma muy importante en la obtención del rendimiento excelente.

Por su parte, los alumnos con rendimiento excelente estudiados en dos muestras españolas muestran alto interés por el estudio al que dedican buena parte de su tiempo disponible, asumen la responsabilidad del propio aprendizaje, tienen cierta conciencia de su capacidad, respetan y son respetados por sus compañeros y profesores, han elegido carreras duras como las ingenierías en mayor medida que la población general, preceden mayoritariamente de la clase media aunque también hay alumnos de la clase social baja y han estudiado predominantemente en centros públicos, entre otros. Por ejemplo el grupo con Premio Extraordinario en Bachillerato destaca en afición lectora, en otorgar un lugar prioritario a las obligaciones escolares, en el dominio de idiomas y de la informática, en valorar la autonomía y responsabilidad personal. Las prioridades académicas, la concentración de esfuerzos, las expectativas, las estrategias y hábitos de trabajo intelectual desarrollados por el alumno figuran entre los factores responsables del rendimiento excelente.

Proceden de familias conservadoras o liberales que coinciden en haber desarrollado en su seno estilos educativos democráticos y coherentes, cuidando el clima familiar 
y las normas que rigen la convivencia. Por ejemplo, desde la infancia los padres han respondido con sencillez a las preguntas de los hijos, han fomentado sus logros, han sembrado expectativas en los hijos y hacia los hijos, han dado importancia al esfuerzo y a la determinación personal y han valorado el buen rendimiento.

Chicos y chicas consideran que, en general, tanto en la escolaridad obligatoria como en el instituto y en la universidad, han recibido un tratamiento equitativo. A pesar de ello si encuentran en ocasiones un tratamiento discriminatorio hacia las chicas, procedente tanto de los profesores como de los compañeros y de la propia familia. Abundan en ejemplos tanto los alumnos con Premio Extraordinario de Bachillerato como los alumnos con Beca de Excelencia, apreciándose en esto últimos mayor discernimiento en el análisis de los hechos.

Alumnos, padres y profesores coinciden al concluir de modo independiente que la escuela no atiende adecuadamente a los alumnos más capaces sino que se limita a repetir, repetir y repetir, y que pese al avance producido hacia la igualdad, perviven estereotipos, prejuicios y actitudes que discriminan doblemente a las chicas dada su condición de mujeres y de mujeres con capacidad. El avance hacia la igualdad ha sido mayor entre la población más joven y más ilustrada y en los ámbitos más cultos y abiertos como la escuela, la universidad y las ciudades, pero aún en ellos persisten reductos machistas soterrados bajo lo "políticamente correcto" y aparecen nuevos estereotipos o viejos estereotipos con nuevos formatos. Coinciden igualmente al señalar que la capacidad cognitiva y el rendimiento escolar excelente se perciben como valores positivos en los chicos y en cierto modo también en las chicas, pero pervive con fuerza aquello de "a las muy listas no las quiere nadie", y afloran algunos estereotipos diferenciados en la conducta social. Los chicos serían más nobles, simples y directos; las chicas más "enrevesadas y brujas". El peso de ciertos estereotipos se mantiene hasta en la universidad pues hay profesores que encuentran difícil otorgar la máxima calificación a una chica aunque su rendimiento sea superior; el mejor debe seguir siendo un varón, estereotipo con muchas caras.

\section{Referencias}

INECSE: resultados en España del estudio PISA 2000. Madrid: Ministerio de Educación y Ciencia, 2005.

JIMÉNEZ, C.; AGUADO, T.; ÁLVAREZ, B.; GIL, J. A.; JIMÉNEZ, R. Caracterización de los alumnos con Premio Extraordinario de Bachillerato. Revista de Pedagogía, Bordón, [S.I.], v. 54, n. 2-3, p. 383-398, 2002.

JIMÉNEZ, C.; ÁLVAREZ, B.; GIL, J. A.; MURGA, M. A.; TÉLLEZ, J. A. Educación, capacidad y género. Revista de Investigación Educativa, [S.I.], v. 23, n. 2, p. 391-416, 2005. 
JIMÉNEZ, C.; ÁLVAREZ, B.; GIL, J. A.; MURGA, M. A.; TÉLLEZ, J. A. Educación, diversidad de los más capaces y estereotipos de género. RELIEVE (Revista Electrónica de Investigación Educativa), [S.I.], v. 12, n. 2, 1-28, 2006.

JIMÉNEZ, C.; ÁLVAREZ, B.; GIL, J. A.; MURGA, M. A.; TÉLLEZ, J. A.; TRILLO MIRAVALLES, M. P. Hacia un modelo sociocultural explicativo del alto rendimiento y de la alta capacidad I: ámbito familiar. Revista Española de Orientación Psicopedagógica, [S.I.], v. 18, n. 1, p. 31-46, 2007.

JIMÉNEZ, C.; MURGA, M. A.; GIL, J. A.; TÉLLEZ, J.A.; TRILLO, M. P. Hacia un modelo sociocultural explicativo del alto rendimiento y la alta capacidad: ámbito académico y capacidades personales. Educación XXI, [S.I.], v. 13, n. 1, p. 125-153, 2010. Disponible en: <http://www.uned.es/educacionXX1/>. Acceso: 20 feb. 2012.

JIMÉNEZ FERNÁNDEZ, C. Educación familiar y alumnos con alto rendimiento. Revista Española de Pedagogía, [S.I.], v. 64, n. 234, p. 273-299, mayo/ago. 2006.

OCDE. Informe PISA 2003: Aprender para el mundo de mañana. Madrid: Santillana, 2005.

OCDE. Informe PISA 2006: Competencias científicas para el mundo de mañana. Disponible en: <http://www.madrid.org/cs/Satellite?blobcol=urldata \&tblobheader=application\%2Fpdf\&blobheadername1=Content-Disposition\& blobheadervalue $1=$ filename\%3DPISA2006_RESULTADOS_INTERNACIONAL.. df\&tblobkey=id\&tblobtable=MungoBlobs\&tblobwhere $=1220389323885$ ttssbinary=true $>$. Acceso: 21 mayo 2011.

OCDE. PISA 2006: Marco de la Evaluación: Conocimientos y habilidades en Ciencias, Matemáticas y Lectura. Madrid: Santillana, 2006.

PISA. PISA 2006: Science Competencies for Tomorrow's World: Executive Summary. Paris: OCDE, 2007. Disponible en: <http://www.pisa.oecd.org/dataoecd/15/13/39725224. pdf>. Acceso: 07 sept. 2010.

PISA. PISA 2009: results: What makes a School Successful? Resources, Policies and practices. Paris: OCDE, 2010.

SMYTH, W. J. Time and School Learning. In: HUSEN, T.; POSTLHWAIT, T. N. (Ed.). The International Encyclopaedia of Education. Oxford: Pergamon, 1985. p. 5265-5262.

TOP of the Class: high Performers in Science in PISA 2006. Paris: OCDE, 2009. Disponible en: <http://www.oecd.org/dataoecd/44/17/42645389.pdf>. Acceso: 20 jun. 2011.

Recebido em: 29/04/2012

Aceito para publicação em: 11/12/2012 